ISSN: $1130-3743$

\title{
LA GLOBALIZACIÓN DE LA EDUCACIÓN
}

\author{
The globalization of the education
}

La mondialisation de l'éducation

Miguel Melendro Estefanía

Universidad Nacional de Educación a Distancia. Facultad de Educación. Departamento de Teoría de la Educación y Pedagogía Social. C/ Senda del Rey, 7. 28040 Madrid. Correo-e: mmelendro@edu.uned.es

Fecha de recepción: enero de 2005

Fecha de aceptación definitiva: abril de 2005

BIBLID [(1130-3743) 17, 2005, 185-208]

\section{RESUMEN}

La educación del siglo XXI se configura en el intento de formar las actitudes, valores y conocimientos de los ciudadanos de un mundo cada vez más cosmopolita. Se perfila como una actividad compleja, implicada en múltiples escenarios antes poco conocidos, cargada de preguntas con respuestas diversas, inciertas, ocupada en la construcción de un modelo humano y social inmerso en el fenómeno de la globalización, escasamente anclado en la necesidad de tomar conciencia de nuestras propias limitaciones.

Establecer el debate en torno a esta idea, desde los argumentos que nos ofrecen las nuevas teorías de las necesidades humanas fundamentales, desde la perspectiva de las herramientas que, hoy, nos señalan cuáles son esos límites del crecimiento humano, y desde el señalamiento de algunos de esos nuevos escenarios educativos, es el objeto de este artículo.

Son diversas las coordenadas desde las que podemos representarnos ese futuro, y cuatro de entre ellas merecen nuestra especial atención: los esfuerzos por educar para la toma de conciencia de los límites de la vida en el planeta; las reflexiones sobre los nuevos espacios sociales -reales y virtuales- de la educación; los escenarios educativos diversos que suponen los países desarrollados y los países en vías de 
desarrollo, centro y periferia del sistema; los enfoques renovados de la educación para la ciudadanía, y el debate actualmente establecido en torno al lugar de la educación entendida como servicio público o como bien de consumo.

Palabras clave: globalización, educación para la ciudadanía, educación virtual, educación ambiental, educación glocal, teorías de las necesidades humanas fundamentales, modelo sinérgico, límites del crecimiento humano, desarrollo sostenible, educación informal.

\section{SUMMARY}

The education of the $21^{\text {st }}$ century is formed in the attempt of forming the attitudes, values and knowledge of the citizens of a world increasingly cosmopolitan. It is outlined as a complex activity involved in multiple scenes before little known, loaded with questions and several uncertain answers and busy with the construction of a human and social model. This model is immersed in the phenomenon of the globalization, scantily anchored in the need to be aware of our own limitations.

The aim of this article is to establish the debate concerning this idea with the arguments that offer us the new theories of the essential human needs, from the perspective of the tools which indicate us the limits of the human growth today, and from some of these new educational scenes.

There are several coordinates to represent that future, and four of them deserve our special attention: the efforts to educate in order to become aware of the limits of the life in the planet; the reflections on the new social spaces -real and virtualof the education; the educational scenes of the developed countries and the developing countries, centre and periphery of the system; the renewed approaches of the education for the citizenship, and the debate established at present concerning the education, about whether it is a public service or a consumer good.

Key words: globalization, education for the citizenship, virtual education, environmental education, "glocal" education, theories of the essential human needs, limits of the human growth, sustainable development, informal education.

\section{SOMMAIRE}

L'éducation du XXI siècle se déroule dans la tentative de former les attitudes, valeurs et connaissances des citoyens d'un monde de plus en plus cosmopolite. Elle se dessine comme une activité complexe, impliquée dans de multiples scénarios peu connus auparavant, chargée de questions avec des réponses diverses, incertaines, occupée dans la construction d'un modèle humain et social immergé dans le phénomène de la mondialisation, faiblement ancré dans le besoin de prendre conscience de nos propres limitations.

L'objet de cet article est celui d'établir le débat autour de cette idée, à partir des arguments que nous offrent les nouvelles théories des besoins humains fondamentaux, à partir de la perspective des outils qui, aujourd'hui, nous indiquent quelles 
sont les limites de la croissance humaine, et à partir de la signalisation de certains de ces nouveaux scénarios éducatifs.

Plusieurs sont les coordonnées à partir desquelles nous pouvons nous représenter ce futur, et quatre parmi elles méritent de notre part une attention spéciale: les efforts pour instruire pour la prise de conscience des limites de la vie dans la planète; les réflexions sur les nouveaux espaces sociaux -réels et virtuels-de l'éducation; les divers scénarios éducatifs que composent les pays développés et les pays en voie de développement, centre et périphérie du système; les nouvelles approches de l'éducation pour la citoyenneté, et le débat actuellement établi autour de l'éducation comprise comme service public ou comme bien de consommation.

Mots clef: mondialisation, éducation pour la citoyenneté, éducation virtuelle, éducation environnementale, éducation glocal, théories des besoins humains fondamentaux, modèle synergique, limites de la croissance humaine, développement soutenable, éducation informelle.

El fenómeno de la globalización se está convirtiendo paulatinamente en un referente cotidiano, en un elemento importante de nuestro escenario vital, y extiende su incidencia a muy diversos ámbitos -económicos, culturales, socialesentre los que el mundo de la educación encuentra también, cada vez más, un lugar destacado.

No resulta exagerado afirmar que la educación está abocada a sufrir serias transformaciones, tanto en sus elementos más inmediatos, didácticos, microsistémicos, como pueden ser los procesos de enseñanza y aprendizaje, hasta los componentes más complejos, estructurales y macrosistémicos, como la propia organización del sistema educativo. Un sistema educativo que, no debemos olvidarlo, hemos construido y conseguido extender a la mayor parte de la población a lo largo del pasado siglo, y que se ha convertido en un referente social, político y en gran medida económico fundamental para las sociedades contemporáneas.

El planteamiento que ha de hacerse de la educación para la vida en un mundo global es mucho más complejo en cierta forma que la idea de educación que, procedente de la "Ilustración", ha impregnado nuestros sistemas educativos actuales. Como afirman Nicholas Burbules y Carlos Torres (2001), "la familia, el trabajo y la ciudadanía, principales fuentes de identificación en la educación de la Ilustración, siguen siendo importantes, ciertamente, pero se están volviendo más efímeras, comprometidas por la movilidad y la competencia con otras fuentes de afiliación".

La educación en un mundo globalizado diversifica cada vez más sus canales formativos: a la influencia de la escuela se suman las posibilidades educativas de los medios de comunicación y de la cultura de la imagen, de otras vías de educación informal y no formal. Una parte de esa educación "global" se encamina, sin embargo, no tanto a la formación de identidades diversas como de identidades globales, cosmopolitas, viables en contextos diferentes, y para ello flexibiliza sus propuestas de forma que sean asequibles a los diferentes entornos culturales, a las 
necesidades cambiantes del mundo del trabajo, a la transmisión a través de los diversos espacios educativos en los que se confunde, cada vez más, formación con información, aprendizaje con consumo... Se abren así posibilidades e incertidumbres, se cruzan una serie de perspectivas que los educadores debemos conocer y debatir, sintiéndonos implicados en esa búsqueda de nuevas identidades que supone la sociedad de la globalización.

Así pues, ¿cuál es el papel de la educación en el intento de formar las actitudes, valores y conocimientos de los ciudadanos de este mundo cada vez más cosmopolita? Iniciar e incitar al debate en torno a esta pregunta es uno de los objetivos del presente artículo.

Algunas de las respuestas fundamentales a la cuestión pueden visualizarse con mayor claridad si nos interrogamos acerca de un tema esencial como es el de las nuevas necesidades creadas en la sociedad del siglo XXI, y las posibilidades, ventajas e inconvenientes de las distintas formas de satisfacerlas. Resultan muy útiles para abordar esta cuestión algunos enfoques que revisaremos a lo largo del texto, como son:

- Inicialmente, el clarificador punto de vista que nos ofrece la nueva Teoría de las Necesidades Humanas Fundamentales, y especialmente la aplicación que desde ellas se hace del denominado "modelo sinérgico".

- La perspectiva que aportan una serie de nuevos instrumentos de medida sobre los límites del crecimiento humano, que ayudan a trazar las fronteras de las interacciones del hombre con el medio ambiente y entre las distintas sociedades humanas, en un mundo cada vez más globalizado.

- Los planteamientos que desde ambas perspectivas confluyen en el concepto de desarrollo sostenible, aquel que procura satisfacer las necesidades de la generación presente sin comprometer la capacidad de las generaciones futuras para satisfacer sus propias necesidades.

Es enorme el potencial educativo que estos planteamientos suponen para la sociedad del siglo XXI, tanto como elementos de reflexión y contraste en torno a los modelos educativos vigentes, como de propuestas de innovación e investigación en diferentes espacios educativos hoy aún poco conocidos. A la caracterización de estos aspectos hace referencia la segunda parte del artículo, en la intención de completar un mapa de los principales retos de la educación para este siglo que viene, desde la perspectiva del fenómeno globalizador. Son diversas las coordenadas desde las que podemos representarnos ese futuro, ya próximo, en ocasiones ya presente, y entre ellas merecen especial atención, desde nuestro punto de vista, cuatro líneas de trábajo: los esfuerzos por educar para la toma de conciencia de los límites de la vida en el planeta; las reflexiones sobre los nuevos espacios sociales -reales y virtuales- de la educación; los escenarios educativos diversos que suponen los países desarrollados y los países en vías de desarrollo, centro y periferia del sistema; los enfoques renovados de la educación para la ciudadanía, y el debate actualmente establecido en torno al lugar de la educación entendida como servicio público o como bien de consumo. 


\section{EL FENÓMENO DE LA GLOBALIZACIÓN}

Como primer paso, conviene definir con la mayor claridad posible el término que marca el punto de partida de este entramado, el concepto de globalización.

El término "globalización" es un término inglés, sinónimo de lo que en francés se denomina "mundialización", y viene a mostrar cómo la sociedad actual es cada vez más la sociedad de la interdependencia, de la proximidad cultural, de la reducción de las barreras geográficas y temporales. Como señala Joaquín Estefanía (2002), una sociedad en la que "nos parecemos más y actuamos de modo crecientemente semejante", en la que se afianza la idea de que vivimos en un mismo y cada vez más reducido mundo.

El concepto de globalización fue acuñado en 1992 por Ronald Robertson, de la Universidad de Pittsburg, para referirse a la intensificación de la conciencia del mundo como un todo, al proceso social por el cual se verifica un retraimiento de las determinaciones geográficas sobre las prácticas culturales y sociales. Por su parte Anthony Giddens (1994), profesor de sociología en Cambridge, ha definido también la globalización como la "intensificación de las relaciones sociales en todo el mundo, por la que se enlazan lugares lejanos, de tal manera que los acontecimientos locales están configurados por acontecimientos que ocurren a muchos kilómetros de distancia o viceversa".

Junto a estas apreciaciones acerca de lo que es y supone la globalización, es importante también dilucidar el significado de otros dos conceptos que con mucha frecuencia la acompañan, y cuyo uso indiferenciado puede inducir a la confusión: los conceptos de globalismo y glocalización. Con el término globalismo se hace referencia a la ideología de la globalización, según la cual todos los problemas pueden resolverse en el mercado global; es la expresión que precisa cómo se ha extendido la economía de mercado a nivel planetario. Con el término glocalización (Robertson, 1992), como veremos más adelante de importantes connotaciones en el ámbito educativo, se quiere hacer referencia a una visión dialéctica de la globalización en relación a la perspectiva local, que hace hincapié en un sentido nuevo del espacio, de los "lugares" del planeta, y que parte de la idea de que no se puede entender la globalización sin el concepto de lo local, de lo próximo y cotidiano.

La globalización, el globalismo y la glocalización se manifiestan a través de una serie de acontecimientos, peculiares e interconectados, que vienen a indicar cómo se están construyendo las nuevas estructuras del nuestro sistema vital, cuáles serán las propiedades emergentes de la sociedad del siglo XXI. Entre ellos podemos citar como más relevantes, y sin ánimo de ser exhaustivos, los siguientes:

- La mundialización de la economía ${ }^{1}$, cuyas características básicas son la interdependencia cada vez mayor de las economías de los distintos países, el incremento de las transacciones internacionales de bienes y

1. El Fondo Monetario Internacional (FMI) plantea una de las ideas más extendidas acerca de la globalización, como es la de su dependencia directa de las estructuras económicas. Ignacio RAMONET (2000) nos la presenta, en este sentido, como "la segunda revolución capitalista". 
servicios, junto a los flujos crecientes de capital ${ }^{2}$. Y, como consecuencia de todo ello, el tránsito del poder político al poder comercial del mercado global único, de forma que los Estados estarían siendo sustituidos, en importantes espacios de toma de decisiones económicas y sociopolíticas, por las empresas, organismos y entidades transnacionales.

- La revolución tecnológica, caracterizada por diversos acontecimientos que hacen notablemente peculiares nuestras sociedades del siglo XXI: la informatización generalizada de los sectores productivos y de la vida cotidiana; la revolución en las comunicaciones a través de la red virtual; la reducción de las distancias geográficas con el uso masivo de los nuevos medios de transporte, y otros no menos significativos e impactantes como pueden ser los grandes avances de la medicina y la atención sanitaria, con sus efectos sobre la situación demográfica del planeta, o las implicaciones que para un futuro no tan lejano tienen los nuevos descubrimientos en el ámbito de la biotecnología.

- La tendencia a la homogeneización cultural, caracterizada por la pérdida de diversidad cultural que revela la progresiva desaparición de antiguas culturas en el planeta ${ }^{3}$, acentuada por los mensajes cada vez más uniformadores difundidos desde los medios de comunicación de masas y la publicidad, e influida, en un sentido aún poco definido, por el mestizaje cultural que suponen los diversos y amplios movimientos migratorios iniciados en el pasado siglo.

- La cultura del "trabajo frágil", en la que disminuyen las posibilidades de disponer de seguridad y estabilidad en el empleo, en la que el tiempo se organiza de forma flexible, y en la que muchas veces es necesario trabajar en varios lugares para poder mantener el mismo nivel de vida ${ }^{4}$ (Beck, 1998).

2. Si distinguimos la economía real, la que crea servicios u objetos concretos, la economía que genera empleo, de la economía financiera o de intercambios virtuales, la de los recursos bursátiles, nos encontramos con que la primera representa un $1 \%$ de las transacciones económicas mundiales, mientras que la segunda representa un $99 \%$... «lo que circula en la mundialización son esencialmente órdenes de compra y venta virtuales por las autopistas de la comunicación" (RAmONET, 2000).

3. "La mejor medida de esta crisis es la pérdida de lenguas. A lo largo de la historia han existido unas diez mil lenguas habladas. Hoy, de las a proximadamente seis mil lenguas que se hablan, muchas ya no se enseñan a los niños -en realidad, ya están muertas- y sólo trescientas las emplean más de un millón de personas. En un siglo, la mitad de las lenguas que en la actualidad se hablan en el mundo pueden haber desaparecido... No son hechos aislados, sino elementos de un fenómeno global que sin duda será recordado como uno de los hechos distintivos de este siglo" (WADE, 1999).

4. Este tipo de trabajo se ha multiplicado de forma notable en todos los países en los últimos treinta años. En Alemania, un tercio de la fuerza laboral tiene empleos frágiles; en Estados Unidos y en el Reino Unido, la mitad. Incluso donde todavía existe el ideal del pleno empleo, ya no se trata tanto de la antigua forma de trabajo a tiempo total. 
- La "Sociedad del riesgo" 5 , a la que se incorporan en clave de complejidad las amenazas actuales a nuestra subsistencia, desde las manifestaciones más violentas del terrorismo internacional a las nuevas enfermedades (SIDA, "vacas locas"...) o las catástrofes ecológicas asociadas a la crisis ambiental, todas ellas de alcance global.

- La creciente convivencia de diferentes modelos de organización mundial: por una parte los Estados nacionales y las relaciones entre ellos; por otra el protagonismo creciente de las organizaciones transnacionales (económicas, como las grandes multinacionales, o sociales, como las organizaciones no gubernamentales) y los organismos intergubernamentales (Banco Mundial, Fondo Monetario Internacional, Organización Mundial del Comercio...). Y todo ello sin olvidar el papel clave en esa organización global de la superpotencia hoy dominante, los Estados Unidos.

Todo ello nos conduce, con argumentos razonables, a respaldar la idea de que vivimos en un mundo difícilmente predecible, donde las consecuencias de las decisiones - de los gobiernos, de las empresas, de los individuos- son cada vez más difíciles de anticipar, donde la incertidumbre es parte de nuestra vida, de la esencia de nuestras sociedades avanzadas, a veces vertiginosa y peligrosamente avanzadas.

2. SOBRE LAS NECESIDADES hUMANAS Y LAS FRONTERAS DEL SISTEMA COMO ÁMBITOS DE REFLEXIÓN EDUCATIVA

La perspectiva que nos ofrece la nueva Teoría de las Necesidades Humanas Fundamentales nos ayuda a recuperar parte de esa seguridad perdida, de la incertidumbre que provoca el sentimiento de transitoriedad ${ }^{6}$, de cambio permanente en nuestro mundo cada vez más globalizado, y nos brinda una vía interesante de reflexión, un camino factible para acometer las actuaciones -también las educativasmás pertinentes, más necesarias.

5. La "Sociedad del riesgo" es para Ulrich BECK (1998), una coyuntura en la que muchos ciudadanos se manifiestan perplejos por la deslocalización de catástrofes que les afectan sin esperarlo y a grandes distancias del epicentro del problema. Beck acuña por ejemplo el eslogan de "Chernobil económico", para describir la dimensión esencial de la sociedad de riesgo global que suponen unas corrientes financieras dificilmente controlables, con capacidad para destruir países enteros.

6. Como señala Henri Bouché (2003), los avances de nuestra sociedad actual «llegan a trascender nuestra capacidad de aprehensión, tanto por su número como por su hondo y complejo significado. La cantidad de información que recibe el ser humano sobre acontecimientos científicos, tecnológicos y de otro orden es capaz de sobrepasar su capacidad de asimilación. Es lo que ha venido en llamarse el "principio de transitoriedad" en virtud del cual hay una estrecha e inversa relación entre la velocidad del cambio y la rapidez limitada de la reacción humana”. 
El enfoque que nos ofrece esta teoría, desarrollada por Antonio Elizalde, Manfred Max-Neef y Martin Hoppenhayn (2001), va más allá de los planteamientos que sobre este tema hicieron otros sociólogos y filósofos, desde F. Engels hasta A. Maslow o A. Héller, al considerar aquéllos como un error muy extendido la creencia de que las necesidades humanas tienden a ser infinitas, que cambian constantemente y varían de una cultura a otra, que son diferentes en cada periodo histórico.

Nos vienen a mostrar estos autores cómo solemos confundir nuestras necesidades, que son pocas, delimitadas y clasificables, las mismas en todas las culturas y en todos los periodos históricos, con las muy variadas formas de satisfacerlas.

Hemos planteado en nuestra teoría que las necesidades son pocas, finitas y consecuentemente pensamos que son clasificables. A nuestro entender existen nueve necesidades humanas fundamentales las cuales serian las siguientes: subsistencia, protección, afecto, entendimiento, creación, participación, ocio, identidad y libertad. Lo que cambia a través del tiempo y de las culturas es la manera o los medios utilizados para la satisfacción de esas necesidades (A. Elizalde et al., 2001).

En este sentido el cambio cultural no será consecuencia de la modificación de las necesidades fundamentales, sino de la mutación más o menos radical de los "Satisfactores" tradicionales de esas necesidades, que serán reemplazados por otros nuevos, diferentes. Estos "Satisfactores" no se corresponden únicamente -ni siquiera primordialmente- con los bienes de consumo disponibles, sino que están referidos a todo aquello que, por representar formas de "ser, tener, hacer y estar", contribuye a la realización de las necesidades humanas. Así, por ejemplo, una de las nueve necesidades humanas fundamentales reseñadas por Elizalde, la de entendimiento", se viene a satisfacer a través de actividades como la investigación, el estudio, la experimentación, la educación, el análisis, la interpretación... actividades que incorporan muy diversos componentes, entre los que el consumo de bienes o servicios no ocupa un lugar especialmente relevante.

El valor que los bienes de consumo pueden tener como satisfactores es limitado, entre otras razones por la forma en que dichos bienes son entendidos como elementos del sistema que sufren procesos entrópicos ${ }^{7}$. Señala A. Elizalde (2001) esa dimensión entrópica de los bienes de consumo al plantear cómo por más que creamos que los procesos económicos terminan exclusivamente en los bienes, eso es falso, ya que en algún momento esos bienes se traducen en chatarra, terminan en lo que es fundamentalmente producción de basura, factor que hoy en día constituye un importante indicador del nivel de riqueza. En la medida en que aumenta el ingreso per cápita y por lo tanto el consumo, aumenta la cantidad -y varía también la composición- de los desechos generados: los pobres, en este sentido,

7. Entropía es la magnitud que nos permite medir la energía que se degrada en los procesos que mantienen vivo todo sistema abierto, y expresa asimismo su mayor o menor tendencia al desorden en un momento dado (Novo, 1995). 
producen poca basura y los ricos producen mucha basura. Tomando este indicador, se puede comprobar cómo los países desarrollados superan los dos kilos de basura diaria por persona y los países subdesarrollados están en torno al medio kilo por persona.

Esto, en cualquier caso, significa que las magnitudes son enormes cuando extrapolamos los datos a los millones de personas que habitan el planeta, y que los procesos entrópicos mencionados necesariamente deben implicar la presencia de unos límites que la producción y el consumo de bienes deben respetar y no pueden transgredir sin que ello suponga serias repercusiones para el equilibrio del sistema, para nuestras condiciones ambientales y sociales de existencia en el planeta.

No en vano estaríamos hablando de una nueva necesidad, en anteriores épocas interpretada y satisfecha de forma bien distinta, y hoy claramente relacionada con la necesidad de subsistencia: la necesidad de tomar conciencia de los límites de la vida humana en el planeta. Trazar estos límites, y aproximar sus actuales "Satisfactores" es tarea que ya hace tiempo se plantea como urgente, y a la que también se debe otorgar la calificación de imprescindible.

\section{APRENDER A TRAZAR LOS LÍMTTES, UNA RESPONSABILIDAD EDUCATIVA Y UN RECURSO DIDÁCTICO}

Encontramos así planteada una de las cuestiones a las que nos referíamos inicialmente: la necesidad de redefinir las fronteras, los "limites" que la vida en un sistema cada vez más globalizado nos impone.

La caracterización de esos límites se ha venido haciendo, con distintas finalidades, desde antiguo; así, el cálculo del Producto Interior Bruto (PIB) ${ }^{8}$, constituyó un primer instrumento de medida útil para contrastar el crecimiento entre los países o regiones del mundo, y un factor esencial para poder avanzar posteriormente en el conocimiento de los límites de ese crecimiento.

El conocido PIB se construye a través de un entramado de indicadores económicos que aportan una estimación cuantitativa de la realidad, pero cuyo alcance se ve limitado ya que opera sobre valores medios y relega la incorporación de los aspectos más cualitativos, que ayudarían a explicar la diversidad y complejidad del contexto social y económico. De esta forma quedan ocultas muchas de las contradicciones y desequilibrios internos de los grupos y comunidades sociales a los que se pretende reflejar. De la interpretación de la realidad formulada con el PIB, que analiza el crecimiento por el crecimiento en sí mismo, se produce el tránsito a una interpretación que viene a centrarse en los límites de ese crecimiento.

Ya a principios de los años setenta, el Club de Roma, junto a un grupo de científicos del Massachussets Institute of Technology, sorprendió al mundo hablando

8. El Producto Interior Bruto (PIB) es uno de los principales conceptos económicos y representa, de forma global, el resultado final de la actividad productiva en una economía; es el valor de los bienes y servicios finales generados por una economía en su territorio. 
de "los límites del crecimiento" (Meadows et al., 1972). Con ello estos científicos se referían fundamentalmente a la idea de que si las tendencias de crecimiento de la población mundial, industrialización, contaminación, producción de alimentos, y explotación de recursos continuaban sin modificaciones, los límites del crecimiento en nuestro planeta se alcanzarían en algún momento "dentro de los próximos cien años; el resultado más probable será una declinación súbita e incontrolable tanto de la población como de la capacidad industrial".

Esos límites del sistema han dado en interpretarse desde muy distintos parámetros $^{9}$. En este sentido, los planteamientos más relacionados con la nueva Teoría de las Necesidades Humanas Fundamentales aportan el enfoque del modelo «sinérgicon ${ }^{10}$ : aquel a través del que, al satisfacer una necesidad determinada, se estimula y contribuye a la satisfacción simultánea de otras necesidades. A través de la puesta en práctica de los principios del modelo sinérgico, se daría respuesta a la satisfacción de nuestras necesidades fundamentales sin perder de vista los límites -referidos o intuidos- del sistema que soporta la vida en el planeta.

La lectura de los límites desde planteamientos novedosos como éste, incorpora instrumentos diversos, de utilización didáctica muy interesante por la relación que establecen entre nuestras actividades cotidianas y los umbrales de crecimiento del planeta, de forma que ayudan a aproximar, desde lo más cercano y vital, algunos de los aspectos del proceso de globalización más teóricos y alejados de nuestra realidad. Entre ellos podemos destacar:

- $\quad$ El IDH (Índice de Desarrollo Humano), del Programa de Naciones Unidas para el Desarrollo, indicador que mide el logro medio de un país en lo referente al desarrollo humano de sus habitantes, desde tres dimensiones básicas: una vida larga y saludable (determinada, sobre todo, por la esperanza de vida al nacer), los conocimientos a los que accede la

9. Luis Alfonso ARANGuREn (2000) se refiere, por ejemplo, a lo que él denomina los “límites de la solidaridad" en los siguientes términos: "existe lo que yo llamo la solidaridad por necesidad. Nace del discurso del Club de Roma que viene a decirnos que si somos inteligentes habremos de ser solidarios, porque en ello nos va la vida... Se trata de la solidaridad resultante de la conciencia del límitem. Merecen también mención relevante los recientemente formulados "límites del Estado-nación", que afirman que los Estados están siendo sustituidos en importantes espacios de toma de decisiones económicas y sociopolíticas por los organismos y entidades transnacionales. Se menciona así un marco político de producción global en el que las empresas multinacionales pueden, por ejemplo, distinguir perfectamente entre lugar de inversión, lugar de producción, lugar de declaración fiscal y lugar de residencia; se trata, por tanto, de la posibilidad de residir allí donde es más atractivo y pagar los impuestos allí donde resulta menos gravoso. En otras palabras, el desarrollo económico queda fuera del control del Estado, que pierde una parte importante de sus ingresos mientras las problemáticas sociales -paro, migración, pobreza- continúan nutriéndose de las arcas de ese mismo Estado. Los límites del Estadonación se transforman así en los límites al modelo de sistema social y político en que nos hemos desenvuelto desde inicios de la revolución industrial.

10. La sinergia es definida, en Teoría de Sistemas, como la acción concertada de varios elementos para realizar una función. 
población (el logro educacional, la alfabetización de adultos y la tasa bruta de matriculación primaria, secundaria y terciaria combinada) y el nivel de vida (el PIB real per cápita). El IDH atiende, pues, no sólo al aspecto económico del desarrollo, sino a las posibilidades reales que ofrecen los países a sus habitantes en cuanto a las oportunidades de educación, la atención médica, la seguridad alimentaria y el empleo.

- En la línea de trabajo del IDH, pero centrado en el ámbito educativo, se propone otro tipo de indicador: el IDE (Índice de Desarrollo Educativo), que clasifica a los países en función de su rendimiento en tres áreas: matriculación en la escuela, terminación de estudios y equidad entre sexos (Arias, 2000). Este indicador a su vez relaciona los datos educativos con el nivel de ingresos del país analizado. Así, puede comprobarse que existen países con serias dificultades económicas que sin embargo alcanzan unos logros educativos muy superiores a los de otros con un mejor nivel de ingresos. China, Cabo Verde, Sri Lanka, Vietnam o Zaire son un claro ejemplo de ello; por el contrario, Arabia Saudí, Colombia o Kuwait son ejemplos de países con un IDE muy inferior al que les correspondería por su nivel de ingresos.

- La huella ecológica es otro instrumento de medida de los límites del crecimiento, y nos ofrece información sobre el espacio biofísico productivo necesario para suministrar los recursos y absorber los residuos generados por una determinada población en su entorno, medido en unidades de superficie equivalentes a la hectárea productiva por persona ${ }^{11}$. Útil para medir el impacto ambiental global, constata las diferencias entre mundo desarrollado y subdesarrollado: Asia, por ejemplo, sólo contaría con un $33 \%$ de superficie productiva del planeta para un $60 \%$ de la población mundial. Actualmente la huella ecológica media mundial (2,85 hectáreas por persona) excede en un $30 \%$ a la media de espacio biológicamente productivo disponible ( 2 hectáreas por persona). Se ha calculado en este sentido que la biosfera necesita seis meses para renovar lo que la humanidad consume en un año, lo que indica claramente que el capital natural de la Tierra se está agotando. Otra comprobación interesante es la de que el impacto de los países ricos cuadruplica al de los países en desarrollo: mientras la huella ecológica de un africano es de 1,4 hectáreas, la de un alemán es de 6 hectáreas, y la de un estadounidense de 12,5 hectáreas (Jiménez Herrero, 2001).

- El IPV (Índice Planeta Vivo) finalmente, es un modelo que se basa en el cálculo de las tendencias de pervivencia de las poblaciones de cientos de

11. Los cálculos actuales evalúan el consumo por nación de sesenta categorías de recursos, desde productos primarios a productos manufacturados; se tienen en cuenta también las importaciones y las exportaciones en relación a la producción nacional. 
especies de aves, mamíferos, reptiles, anfibios y peces; indica también claramente cómo la actual presión del consumo humano es poco sustentable. A través de los últimos 30 años, el IPV ha disminuido en un 35\%; la merma en especies de agua dulce ha sido especialmente dramática, con un promedio del 54\% de disminución. Las especies marinas también se encuentran amenazadas, con un promedio de reducción del $35 \%$, y las poblaciones de especies forestales muestran una reducción del 15\%. Según el Informe Planeta $\mathrm{Vivo}^{12}$, a menos que los gobiernos adopten acciones urgentes, para 2030 comenzará a declinar el bienestar humano, medido por la esperanza de vida, el nivel educacional y el producto económico.

\section{Las Claves de UN FUtURO SOSTENIBLE}

Junto a la definición de estos límites, es necesario construir los nuevos satisfactores que nos permitan dar respuesta a la necesidad de subsistencia dentro de ellos; la ya tradicional definición de desarrollo sostenible viene a ayudarnos en ese sentido. Entendido como aquel desarrollo que satisface las necesidades de la generación presente sin comprometer la capacidad de las generaciones futuras para satisfacer sus propias necesidades (CMMAD, 1987), el concepto de desarrollo sostenible establece un peculiar tipo de relación entre las nociones anteriormente trabajadas sobre los límites del crecimiento, las necesidades fundamentales y las formas en que pueden ser satisfechas, clave como hemos visto para interpretar las sociedades humanas del futuro y para perfilar su compleja y vital interacción con el medio ambiente.

Los organismos internacionales han ido apuntando, cada vez con mayor insistencia, hacia el tránsito de una educación ambiental a una educación para la sustentabilidad; considerando el carácter muchas veces reduccionista de la primera, centrada fundamentalmente en actuar sobre los problemas del medio ambiente físico, se ha hecho referencia en diversos foros mundiales -Cumbre de la Tierra (1992), Conferencia sobre Educación y Sensibilización para la Sostenibilidad de Tesalónica (1997), Cumbre Mundial sobre el Desarrollo Sostenible de Johannesburgo (2002)- al papel esencial que la educación debe jugar en la construcción de conocimientos, criterios y comportamientos propios de una sociedad sostenible ambiental y socialmente. Como indican Amparo Vilches y Daniel Gil (2003), "la capacidad de adoptar decisiones fundamentadas exige conocimientos para sopesar las consecuencias a medio y largo plazo; exige criterios para comprender que lo que perjudica a otros no puede ser bueno para nosotros; exige educación". Estos mismos autores subrayan lo escasos que aún son los recursos destinados a la educación para la sostenibilidad, y de qué modo influye en ello el desconocimiento de

12. Realizado en el año 2000 por WWF y avalado por 175 científicos de todo el mundo, se recoge en él información a partir del uso de medidas como la Huella Ecológica y el Índice Planeta Vivo. 
la sociedad acerca de los análisis globales sobre los problemas relacionados con el desarrollo y el medio ambiente, y también el escaso interés y sensibilización mostrado por el conjunto de los educadores hacia estos temas.

Ahondando en estos argumentos y en sus consecuencias educativas, en la Reunión Internacional de Expertos en Educación Ambiental de Santiago de Compostela (UNESCO, 2000) se señalaba la importancia que para una adecuada educación ambiental estaba teniendo la puesta en práctica del conocido lema de los ecologistas alemanes "actúa localmente, piensa globalmente", y se cuestionaba en relación a él la dificultad existente para establecer esa conexión entre lo local y lo global en la práctica educativa, y la necesidad por tanto de revisar la forma en que esa conexión puede ser potenciada, reforzada. En esta línea se plantea una práctica educativa que debe incrementar el análisis global de la realidad, y que ha de orientarse tanto al tratamiento de los problemas locales detectados cómo al fomento de la participación en la búsqueda y puesta en práctica de soluciones a esos problemas, y también a la valoración de las repercusiones que, a nivel local y también global; pueden derivarse de las actuaciones emprendidas.

\section{OtRAS REALIDADES, OTRAS PERSPECTIVAS: LAS DIMENSIONES DE UNA EDUCACIÓN "GLOCAL"}

En relación con todo lo comentado anteriormente, y a la pregunta que inicialmente nos formulábamos sobre cuál debe ser el papel de la educación en un mundo globalizado, habría que responder, por tanto, resaltando la importancia de educar en una serie de actitudes, valores y conocimientos relacionados con los modos, y no tanto con los productos, bienes o servicios, a través de los que la sociedad de la globalización satisface nuestras necesidades fundamentales. Este enfoque sobre formas y condiciones, más que sobre recursos y utilidades, encuentra, de modo sinérgico con lo planteado en relación a una educación para la sostenibilidad, un punto de referencia esencial en la propuesta de "pensar globalmente y actuar localmente". Esta propuesta ha sido enriquecida recientemente con las aportaciones de quienes, como Ronald Robertson, han reflexionado acerca de sus posibilidades para ser llevada a la práctica. Así, Robertson introduce un nuevo término que relaciona lo global y lo local, el término "glocal", y define el proceso de "glocalización" (Beck, 1998) como una secuencia que supone el cambio en la organización de los Estados, de las naciones, hacia una nueva estructura de toma de decisiones que puede interpretarse encauzada en tres direcciones:

- Hacia dentro, hacia la participación de la propia sociedad que constituye el Estado en la determinación de sus metas y logros.

- Hacia fuera, en relación con las nuevas redes de decisión y puntos nodales de los escenarios políticos transnacionales. 
- Y hacia abajo, hacia lo local, en un proceso de localización de lo global y de globalización de lo local que contrasta con el planteado por Prebisch en su conocida teoría del centro y la periferia ${ }^{13}$.

En lo que se refiere a los sistemas educativos ${ }^{14}$, el sentido de este proceso de glocalización supone también una serie de tendencias:

- Hacia la participación de la sociedad en la toma de decisiones sobre su propio futuro, utilizando los nuevos espacios sociales de la educación -la educación virtual, la educación no formal e informal, interactuando de forma peculiar con la educación reglada- y recuperando las propuestas de "sociedad educativa" ya formuladas el pasado siglo. Se trata aquí de abordar, desde el ámbito educativo, ese carácter endógeno, "hacia dentro", descrito por Robertson en el proceso de glocalización.

- Educando, esta vez en sentido exógeno, para la comprensión de los escenarios globales y las nuevas redes de relaciones que en ellos se tejen. Como paso previo, formando desde el conocimiento, la comprensión y la toma de conciencia de los límites del sistema en el que vivimos, y planteando simultáneamente un modelo de sociedad y de educación "sostenibles" que actúe sobre las emergencias del sistema, aquellas de sus cualidades que sin estar localizadas específicamente en alguno de sus componentes tienen una importante capacidad de transformar el sistema mismo. Estamos aquí hablando de una educación glocal "hacia fuera", desde la frontera más o menos nítida entre lo próximo y lo global.

- Provocando, por último, la reflexión sobre los desequilibrios de la educación a nivel local -entre las sociedades hoy dominantes y sus periferias- y sobre la mundialización educativa, que aborda en este momento el crucial debate sobre la educación entendida como servicio público o su consideración como bien de consumo.

13. Según la Teoría del Centro y la Periferia, enunciada por R. PREBISCH (1976), mientras el centro del sistema -constituido por los países más desarrollados- desempeña la función de toma de decisiones en un espacio social, económico y natural muchas veces diferente del espacio en que esas decisiones se ejecutarán, la periferia -constituida por los países pobres y menos desarrollados- aporta recursos humanos y económicos al desarrollo del centro, encontrándose las posibilidades de desarro110 de la periferia determinadas por las condiciones que gobiernan sus relaciones de intercambio con el centro. A su vez se generan en la periferia contenidos y papeles sociales a imagen y semejanza del centro, mientras que desde éste se valora inadecuadamente el impacto ambiental de las decisiones exportadas a la periferia, reforzándose en ambos sentidos la interdependencia existente.

14. El término glocalización es aplicado ya en numerosos ámbitos, desde los nuevos enfoques empresariales hasta campos como la seguridad, el marketing o Internet; multinacionales como Sony, Coca Cola o MSN emplean nuevos términos relacionados con la glocalización, estructuran sus mercados en función de la deslocalización y relocalización de recursos, y se plantean políticas de "localización global". El propio Ulrich $\operatorname{BECK}(1998,78)$ hace referencia a la "glocalización" en el ámbito sociocultural y muestra como ejemplo la situación de los matrimonios y familias transculturales, cada vez más abundantes en las sociedades desarrolladas. 
Si la educación, como proponen Burbules y Torres (2001), es aquella actividad a través de la que nos hacemos conscientes del mundo en que vivimos, de sus posibilidades y también de sus limitaciones, vemos cuán esencial es indagar acerca de los diferentes aspectos que hasta aquí hemos ido describiendo, y lo necesario que resulta aprender a interpretar y a afrontar, desde el ámbito de la pedagogía, este fenómeno de la globalización que implica formas diversas, muchas veces divergentes, de satisfacer nuestras necesidades fundamentales.

Un primer paso para ese "hacernos conscientes" del mundo en que vivimos vendrá apuntado por los nuevos espacios educativos que se van construyendo en la sociedad del siglo XXI, por los cambios que se vislumbran en el ámbito de la pedagogía de mano de la mundialización de la economía, de la revolución tecnológica de la información y las comunicaciones, de la lenta e inexorable homogeneización cultural, sin perder de vista los diferentes contextos en que esos cambios se van implantando, ni las referencias que una reforzada generación de derechos -derechos humanos y del ciudadano, ecológicos, de las diferentes comunidades que habitan el planeta...- nos aportan.

\section{LOS NUEVOS ESPACIOS SOCIALES DE LA EDUCACiÓN}

Si comenzamos abordando, como señalábamos anteriormente, las facetas endógenas, "hacia dentro", del fenómeno de la glocalización en el ámbito educativo, hemos de tomar como referencia las pautas que desde los nuevos espacios sociales de la educación se vienen marcando a los procesos de enseñanza-aprendizaje. Es cierto que en los espacios educativos actuales encontramos transformaciones significativas respecto a épocas anteriores; una de ellas tiene que ver con la gran incidencia de la denominada educación informal -aquella que tiene lugar espontáneamente a partir de las relaciones del individuo con su entorno, y que generando efectos educativos no ha sido planificada pedagógicamente en función de objetivos educativos explícitos-, representada por numerosos e innovadores entornos educativos, que incluyen desde los programas televisivos a los videojuegos, de la elaboración y consulta de páginas web a la participación en grupos de correo electrónico, desde la educación a distancia y el e-learning hasta los significativos cambios que se han producido en la educación en el seno de la familia o en la participación a través de las organizaciones no gubernamentales.

Los medios de comunicación de masas, y en concreto la televisión, son uno de los componentes más activos actualmente como soportes de educación informal. Una reciente investigación del grupo T.N. Sofres (2003) señala, por ejemplo, cómo los niños españoles están una media de 218 minutos diarios ante la pantalla de televisión, frente a los 300 minutos que pasan en sus centros educativos ${ }^{15}$. Es

15. Investigación del Grupo "Taylor Nelson Sofres" sobre audiencia infantil en las televisiones españolas para el Ministerio de Educación, publicada en el diario El País del 6 de noviembre de 2003. Se puede consultar información complementaria en www.ceaccu.org. 
cada vez más notoria la influencia de las nuevas tecnologías en la transformación de los sistemas educativos; diversos, novedosos y en ocasiones contradictorios roles son desempeñados por educadores y educandos como resultado de las variadas interconexiones que se producen entre los diferentes espacios educativos hoy posibles.

La Sociedad Red, el Tercer Entorno, el Ciberespacio, la Aldea Global ${ }^{16}$, son términos que intentan expresar de forma sintética lo que supone esa nueva realidad. Surgen como metáforas de un medio ambiente virtual, un espacio inmaterial, no físico pero sí real, donde tienen lugar las actividades que se llevan a cabo mediante las nuevas tecnologías de la comunicación y la información" (Murga, 2002), y que constituye uno de los principales escenarios en el que ha de desenvolverse la nueva pedagogía. En nuestros días podemos asistir sin pestañear a eventos que hubieran sido en otro momento extraordinarios, hasta hace poco improbables, como el que supuso el pasado mes de abril de 2003 la "Gran Lección": más de un millón de alumnos participaron en la mayor clase simultánea de la historia. Durante treinta minutos, en más de un centenar de países se abordó sincrónicamente y de forma coordinada, gracias a las posibilidades que ofrecen las nuevas tecnologías de la comunicación, el problema de las desiguales oportunidades en el acceso a la educación de hombres y mujeres.

Hoy también nos encontramos con uno de los más graves inconvenientes de la globalización de la información, con la gran contradicción de que resulta más problemático "digerir» la información que nos llega, aprender a asimilarla, que acceder a ella. Los excesos informativos se convierten en un obstáculo, y son de escasa ayuda para entender realmente dónde nos situamos, para ser conscientes de los límites y las posibilidades que nos plantea, y que nos ofrece, el mundo en que vivimos. Se trata del conocido "principio de transitoriedad", en virtud del cual hay una estrecha e inversa relación entre la velocidad de los cambios que se producen en nuestro entorno y la rapidez y la capacidad limitada de la reacción humana para aprehenderlos (Bouché, 2004).

En otra vía de interés primordial en lo que entendemos como procesos de glocalización endógena, esta vez desde el ámbito de la educación no formal ${ }^{17}$, diversos pedagogos y educadores -Ivan Illich y Everett Reitmer entre otros- fueron proponiendo en la segunda mitad del pasado siglo una paulatina apertura a la

16. En el ámbito de las teorías de la comunicación, ya en 1960 Marshall McLuhan había formulado el célebre concepto de la "aldea global" asociado al desarrollo de las comunicaciones electrónicas: "los medios electrónicos del hombre postalfabetizado contraen el mundo hasta reducirlo a una aldea o una tribu en la cual todo le sucede a todo el mundo al mismo tiempo y conceden esta calidad de simultaneidad a los acontecimientos que tienen lugar en esta aldea global.

17. La educación no formal es definida como el conjunto de medios e instituciones que generan efectos educativos a partir de procesos intencionales, que cuentan con objetivos pedagógicos previa y explícitamente definidos, desarrollados por agentes cuyo rol educativo está institucional o socialmente reconocido, y que no forman parte del sistema educativo graduado (Trilla, 1985). 
influencia formativa de la sociedad de los procesos educativos que tradicionalmente se ubicaban en el marco escolar; esta "Sociedad educativa" vendría necesariamente acompañada de una progresiva desescolarización de los procesos de enseñanza-aprendizaje.

En ese sentido, más recientemente el italiano Francesco Tonucci (1997) nos anima también, en su discurso sobre la educación, a romper el monopolio de la escuela, a abrir ésta a su entorno, a construir la "ciudad educativa" de la que ya se comenzó a hablar en los años sesenta y setenta. Su "proyecto educativo integrado", en el que caben la escuela, las organizaciones sociales, el gobierno de la ciudad, las estructuras productivas, las instituciones culturales, la familia y las redes primarias de socialización, y su propuesta de "Ciudad de los Niños", coinciden en numerosos aspectos con los proyectos de Illich y Reitmer, y forman un núcleo que, junto a las posibilidades que ofrecen las nuevas tecnologías, cada vez se acerca más a la idea de una educación "hacia dentro", de síntesis entre lo glocal y lo local, construida desde, para y por la sociedad que la cimienta. La progresiva pérdida del monopolio -en educación- de las estructuras educativas formales -escuela, instituto, universidad- junto a la extensión sinérgica tanto de la educación informal como de la educación no formal, compone un universo con enormes posibilidades para la educación que ha de venir.

\section{EDUCACIÓN CIUDADANA Y CONCIENCIA DE LOS LÍMITES}

Otra característica esencial de esa educación pensada en términos de futuro, tiene que ver con su posicionamiento en sentido exógeno, "hacia fuera", en la interacción entre lo global y lo local que es nota característica de nuestra sociedad de inicio del tercer milenio. Una educación para la comprensión de los escenarios globales, para el conocimiento, la construcción cabal y la participación en las nuevas redes de relaciones que supone el entramado de la mundialización. Podemos observar cómo los objetivos de la educación van mutando, poco a poco, hacia la flexibilidad y la adaptabilidad, hacia el aprendizaje de la coexistencia en espacios diversos -y con frecuencia también divididos por el conflicto-, hacia la construcción de un sentido de identidad que pueda seguir siendo válido en contextos muy diversos. La denominada "educación para la ciudadanía mundial" se está convirtiendo en una forma de canalización de esas expectativas, y se postula cada vez más como uno de los instrumentos capaces de aportar, en las instituciones educativas locales, nacionales y transnacionales y desde una perspectiva "glocal", la conciencia crítica necesaria para dar a conocer y ayudar a tomar decisiones sobre las consecuencias deseadas y no deseadas del fenómeno de la globalización (Imbernon, 2002; Gimeno, 2003).

Señala Gimeno Sacristán cómo la incertidumbre que nos provoca la globalización hace imprescindible sentar unas premisas básicas que nos ofrezcan alternativas para esa educación de futuro. 
En un mundo interconectado, en el que los poderes se ocultan, las razones se explican con lenguajes incomprensibles y las relaciones causa-efecto son difíciles de comprobar, la soberanía popular y el poder del ciudadano para regir los destinos de la sociedad parecen una quimera o un privilegio reservado a unos pocos. Todas esas nuevas circunstancias afectan muy decisivamente a los sistemas educativos, a las políticas que se desarrollan para ellos, a las prácticas educativas y, lo que es más importante, alteran el valor que la educación tiene en sí misma como proyecto de progreso individual y social (Gimeno Sacristán, 2003).

El propio Gimeno Sacristán apunta un listado de propuestas, que él denomina la "carta de la educación democrática del ciudadano", y que atiende a su libertad y autonomía, a la igualdad y la solidaridad, en las coordenadas del acceso a la educación, los contenidos y las prácticas educativas, las relaciones interpersonales y las relaciones de la escuela con la comunidad. Una "carta" para todos los países, global, que debe ser aplicada en función de las necesidades próximas, locales, en un nuevo ejercicio de glocalización.

Como se ha venido planteando a lo largo de este trabajo, esa educación para la ciudadanía debe partir del conocimiento, la comprensión y la toma de conciencia de los límites del sistema en el que vivimos. Retomando las propuestas de la Nueva Teoría de las Necesidades Humanas Fundamentales, recordamos que la construcción de conocimientos, el estudio, la investigación y la educación son algunos de los elementos que aportan satisfacción a la necesidad humana fundamental del "entendimiento" (Elizalde, 2001). Educar para la toma de conciencia de los límites de la vida humana en el planeta vendría a satisfacer no sólo esta necesidad fundamental de "entendimiento", sino también otras necesidades fundamentales como la de "Subsistencia" o la de "protección", en ese juego de sinergias a través del que, al satisfacer una necesidad determinada, se estimula y contribuye a la satisfacción simultánea de otras necesidades.

Cómo establecemos esas sinergias es algo que la educación ambiental lleva trabajando desde sus primeros momentos, y que ha ido perfeccionando a lo largo de las últimas décadas. Las elaboraciones didácticas a partir del estudio y aplicación de instrumentos como el Índice de Desarrollo Humano o la Huella Ecológica se han convertido ya en una parte muy importante de la nueva educación ambiental, y multiplican las aportaciones educativas relacionadas con el fenómeno de la globalización. Como señala Michela Mayer (2001), la educación ambiental abre nuevos espacios para producir nuevos saberes, saberes críticos que ayuden a enfrentar el desafío de "construir una globalización responsable y conjugarla con el refuerzo de la identidad local."

En los últimos años hemos asistido también al desarrollo de múltiples iniciativas educativas, la mayor parte de ellas promovidas desde el ámbito de la educación ambiental, que han acercado a la educación a esa conciencia de las fronteras del sistema. Se trata de iniciativas en muchos casos internacionales, que incorporan a miles de centros educativos en el desarrollo de sus actividades. Merece la pena destacar algunas que han dado lugar a redes de intercambio de conocimientos 
y proyectos educativos, conectando lo local con lo global ${ }^{18}$, como pueden ser el Proyecto Ecoescuelas o el Programa GLOBE (Global Learning and Observations to Benefit the Environment ${ }^{19}$. En el primero ${ }^{20}$ participan centros educativos de primaria y secundaria, universidades y otras instituciones educativas, en un proyecto que implica la adecuada gestión ambiental de los centros, junto al tratamiento interdisciplinar de los contenidos relacionados con la educación ambiental, y el diseño y realización de actividades de forma participativa por toda la comunidad escolar. El segundo, que puede traducirse como Programa de Aprendizaje y Observaciones Globales en Beneficio del Medio Ambiente, es un programa destinado a centros de secundaria que promueve la investigación del entorno de los centros y la elaboración de información significativa acerca de las características del ecosistema y del impacto ambiental que soporta, información que es recogida, tratada y posteriormente divulgada a través de Internet.

Son numerosas también las iniciativas que participan de espacios como el de la educación virtual para hacer llegar el mensaje sobre los límites; la construcción de múltiples web-quest es una clara muestra de ello; se trata de unidades didácticas virtuales que ofrecen contenidos, proponen actividades y facilitan la autoevaluación y en general el quehacer educativo sobre muy diversos temas educativos, muchos de ellos relacionados directamente con la educación ambiental y, de manera destacada, sobre huella ecológica e impacto ambiental ${ }^{21}$.

De especial interés resultan todas las actuaciones educativas que confluyen en los programas sobre Agenda 21 Escolar, también de alcance internacional, y que relacionadas con el Programa 21 de la Cumbre de la Tierra (Río de Janeiro, 1992) plantean el diseño y desarrollo de actividades educativas en torno al concepto de desarrollo sostenible, generalmente relacionadas con otras actuaciones de tipo social, ambiental, urbanístico, tecnológico, sanitario... encaminadas a favorecer la sustentabilidad a nivel local o comunitario. De forma sinérgica con estas líneas de actuación, se han constituido en los últimos años redes de Ciudades Sostenibles ${ }^{22}$,

18. "Hoy de la educación se precisa, fundamentalmente, capacidad para enfrentarse a realidades complejas, relacionadas incluso por redes de sistemas, por un reticulado complejo y dinámico, que forma la sociedad actual que, no se olvide, es la sociedad de la globalización, de la mundialización y de las nuevas tecnologías" (COLom, 2003).

19. Información actualizada sobre estas iniciativas puede encontrarse en sus páginas web: http://www.eco-schools.org y http//www.globe.gov.

20. El Proyecto Ecoescuelas, gestionado por la Fundación para la Educación Ambiental (FEE) con el reconocimiento de la ONU, se encuentra actualmente implantado en más de catorce mil centros educativos.

21. Un buen ejemplo es la página www.earthday.net/footprint, a través de la que podemos calcular la huella ecológica personal, nuestra incidencia en el planeta y algunas de las alternativas para modificarla.

22. Las "Ciudades Sostenibles" se constituyen siguiendo los planteamientos de la Agenda 21 Local de la Cumbre de la Tierra (1992), y en Europa se agrupan y organizan como tales a partir de la conocida "Carta de Aalborg o Cartas de las Ciudades Europeas hacia la Sostenibilidad", firmada en 1994 por 80 autoridades locales y 253 representantes de diversas organizaciones. 
Ciudades de los Niños, Ciudades Educadoras ${ }^{23}$ y Ciudades Saludables ${ }^{24}$, que contemplan de forma prioritaria, en su entramado teórico y entre sus actividades prácticas, las propuestas educativas en torno a la conciencia de los límites y la educación para un desarrollo sostenible.

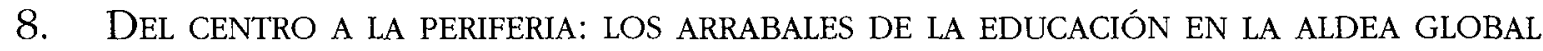

Junto a esta panorámica de los procesos de cambio, que se siguen fundamentalmente en los sistemas educativos del mundo occidental, del Norte desarrollado, se encuentra la perspectiva de los riesgos que supone la expansión del enfoque "globalista", así denominado por Ulrich Beck $(1998)^{25}$, a otros lugares del planeta.

Uno de ellos es el riesgo de interpretar "nuestro mundo" como el «único mundo" posible o deseable. Desde las ONGD (Organizaciones No Gubernamentales para el Desarrollo) no dejan de señalarlo, sacando a la luz situaciones cuya existencia sería inimaginable en "nuestro" Norte, y que pueden fácilmente vislumbrarse a partir de algunos de los siguientes indicadores:

- Numerosos países africanos registran tasas de escolarización decrecientes... y un notable incremento del trabajo infantil.

- Las diferencias educativas entre el mundo rural y el urbano son cada vez más profundas. En países como Perú, las tasas de analfabetismo en áreas rurales triplican a las de las ciudades; en otros, como por ejemplo Zambia, el $50 \%$ de la población rural vive a más de 10 kilómetros del colegio más cercano.

- Casi seiscientos millones de mujeres en todo el mundo son analfabetas, a pesar de que es conocido que la educación de las mujeres puede tener una enorme influencia en la reducción de la mortalidad infantil, el

23. Las «Ciudades Educadoras", agrupadas en la Asociación Internacional de Ciudades Educadoras (AICE), parten de una propuesta integradora de la vida ciudadana que concierne a gobiernos locales, pero también a todo tipo de instituciones y asociaciones públicas y privadas. Su objetivo es trabajar conjuntamente con sentido educativo en el desarrollo de políticas y actuaciones que impulsen la calidad de vida de las personas, su compromiso con el espíritu de ciudadanía y los valores de una democracia participativa y solidaria.

24. El Proyecto "Ciudades Saludables" fue promovido por la OMS a partir de 1987, y en la actualidad más de 50 naciones poseen su propia red nacional de ciudades saludables, con unos desarrollos estratégicos coincidentes con los de las ciudades sostenibles y educativas, especialmente en relación a sus planteamientos en torno a la equidad, el desarrollo social y el desarrollo sostenible.

25. El globalismo, para Beck, es la ideología de la globalización, y viene a plantear que todos los problemas pueden resolverse en el mercado global; el predominio de la economía de mercado a nivel mundial relega a un plano secundario los fundamentos del autodesarrollo democrático. Y como tal, opina Beck, es uno de los mayores riesgos para el establecimiento de una cultura democrática. 
control de la natalidad y, en general, en la mejora de la salud de la población, junto a otros aspectos sociales y culturales.

- En el África Subsahariana y América Latina los salarios de los profesores han descendido en un tercio en términos generales desde mediados de los años ochenta, y el absentismo de los profesores por esta causa, entre otras, se ha convertido en uno de los grandes problemas educativos en muchos países.

- La crisis económica de mediados de los noventa en el sudeste asiático ha provocado que millones de niños -en países como Tailandia o Indonesia- hayan dejado de asistir a la escuela al aumentar el precio medio de los cuadernos escolares, los zapatos o el transporte (Arias, 2000).

Todo esto sin olvidar que también aquí, en nuestro Norte, cohabitamos con un Sur que igualmente es "nuestro", y que representa la marginación y la pobreza. Se trata del que se viene denominando Cuarto Mundo, el mundo de subdesarrollo que subsiste en el seno del "mundo desarrollado". Un Cuarto Mundo que ha de hacernos reflexionar, aunque sólo sea porque en él se reflejan los efectos de la peor de las miserias, la de quienes viven rodeados de una abundancia a la que no pueden acceder, la de quienes pueden ver de cerca el despilfarro irresponsable sintiendo insatisfechas sus necesidades más primarias.

El reciente fenómeno de la inmigración ha venido, en parte, a modificar las características de ese "Sur en el Norte", no sólo sufriendo las consecuencias de la injusticia y la falta de equidad, sino también aportando las posibilidades de una riqueza e intercambio cultural sin precedentes; no en vano sólo la creciente inmigración ha sido capaz de incrementar, por primera vez en quince años, el número de estudiantes en la educación primaria de nuestro país.

En ese contexto, una de las consecuencias que las pujantes políticas en defensa de una economía de mercado vienen planteado a nivel global, y que sin duda afectará de forma importante a esa diferencia entre centro y periferia, entre norte y sur del planeta, es la propuesta de reducción de las inversiones públicas en temas sociales, potenciando la privatización y la aplicación, también en los ámbitos educativos, de las teorías de gestión y organización empresarial.

En este sentido, tanto por parte del Foro Mundial de la Educación (FME) ${ }^{26}$, como por parte de la Internacional de la Educación (IE) ${ }^{27}$, se ha manifestado la oposición a una posible mercantilización de la enseñanza y más concretamente al proyecto de la Organización Mundial del Comercio de liberalizar los servicios, incluidos los educativos, a través del nuevo AGCS (Acuerdo General de Comercio y Servicios), que se encuentra en proceso de inminente aprobación.

26. Celebrado en la ciudad de Porto Alegre el año 2003 con motivo del Foro Mundial Social.

27. La Internacional de la Educación es una organización mundial de profesionales de la educación, integrada por 24,5 millones de miembros que representan a todos los sectores de la educaciôn, del preescolar al universitario, a través de 309 organizaciones de 157 países y territorios. 
La educación, y especialmente la educación superior, están ya insertas en un fuerte proceso de privatización. Se puede observar además, cada vez con mayor frecuencia, cómo en las instituciones educativas se introducen fórmulas de patrocinio e influencia económica -que deviene en ideológica- por parte de las grandes empresas transnacionales, a través del ejercicio del mecenazgo en centros escolares y universidades, e incluso en movimientos sociales que promueven la educación ambiental o la solidaridad con el Tercer Mundo.

Uno de los objetivos tradicionales y prioritarios de la educación, la preparación para el mundo del trabajo, se está viendo también afectado en este sentido por los efectos de la globalización sobre la formación y el empleo. Las instituciones educativas se ven en la necesidad de adaptarse a un mercado de trabajo cambiante, aportando nuevas calificaciones, flexibilizando los currículos, y también, en un mercado paulatinamente más competitivo, implantando cada vez más las evaluaciones de rendimiento, exámenes o itinerarios selectivos, que vienen a ordenar la "reserva" de mano de obra internacional (Chomsky, 1998).

\section{UN ESBOZO PARA EL FUTURO: A MODO DE CONCLUSIÓN}

Hemos recorrido los complejos itinerarios de un mundo cada vez más globalizado, más común a todos nosotros, pero no por ello menos rico y diverso aún. Como bien se sabe en las ciencias ambientales, la pervivencia y la salud de un ecosistema depende en gran medida de su diversidad, del equilibrio entre sus componentes, de las posibilidades reales de retroalimentación que se pueden establecer ante acontecimientos críticos, antes situaciones emergentes que vienen a decantar su futuro en uno u otro sentido.

El mundo que conocemos, en el que ahora habitamos, es un gran sistema en el que la vida, y especialmente la vida de los seres humanos, ha alcanzado sus límites de crecimiento ante lo que se puede entender como un riesgo para la supervivencia. Precisamente esa necesidad vital, la de subsistir, nos obliga a plantear la importancia de educar para que todos lleguemos a entender los límites del planeta y de la vida sobre él, tal y como la conocemos. Unos límites que nos abocan a buscar nuevas formas de satisfacer nuestras necesidades fundamentales -cuánto más las superficiales y prescindibles- considerando, en primer lugar, lo sustentable o no de nuestros actos, de nuestras decisiones, en relación a las posibilidades reales de desarrollo en el planeta.

La educación tiene un importante papel en este empeño, y unas obligaciones que dimanan de él. Transmitir y provocar aprendizajes significativos, permanentes, en la población del planeta, sobre esos nuevos contenidos formativos; hacerlo en función de unos planteamientos éticos que respondan a las nuevas formas de satisfacer nuestras necesidades, al modelo sinérgico que las fortalece; construir propuestas educativas adaptadas a los nuevos espacios de la educación que ya están marcando nuestras vidas, y ayudar a conseguir el cambio necesario en las formas 
de hacer, de pensar y de vivir en ese mundo global. Un mundo global que no debe seguir consintiéndose la triste e insostenible globalización de la pobreza, de la ignorancia, del deterioro del medio ambiente o de la violencia.

Con las limitaciones que la incertidumbre incorpora a toda propuesta, máxime cuando se navega en el rumbo de lo complejo, éste puede ser un punto de arranque en el debate sobre la educación que nos viene. Es quizás prioritario, en estos momentos de cambio casi compulsivo, apremiante, plantear bien las preguntas, comprender el contexto en el que y sobre el que se formulan. Y también lo es buscar respuestas compartidas a esos problemas cada vez menos lejanos, más comunes y próximos, aportar la réplica sensata a esas situaciones que, sin dejar de repetirse, no dejan aún de sorprendernos.

\section{BIBLIOGRAFÍA}

AA.VV. (2003) Atlas de Le Monde Diplomatique. París, SA Le Monde Diplomatique.

ARANGUREN, L. A. et al. (2000) El proceso de globalización mundial. Barcelona, Intermón.

ÁRIAS, M. (2000) Educación abora. Barcelona, Intermón.

ARTHUR, J. y BAILEY, R. (2000) Schools and community. Londres, Falmer Press.

BECK, U. (1998) ¿Qué es la globalización? Madrid, Paidós.

BOUCHÉ, H. (2003) Educar para un nuevo espacio bumano. Madrid, Dykinson.

BURBULES, N. y TORRES, C. (2001) Globalización y Educación, Revista de Educación, Número extraordinario.

CARPENTER, E. y McLUHAN, M. El aula sin muros. Investigaciones sobre técnicas de comunicación. Barcelona, Ediciones de Cultura Popular.

CASTEL, O. (2002) Le Sud dans la mondialisation. París, La Découverte.

CHOMSKY, N. y HeInZ, D. (1998) La Aldea global. Tafalla, Txalaparta.

CMMAD (Comisión Mundial del Medio Ambiente y del Desarrollo) (1987) Nuestro Futuro Común. Madrid, Alianza Editorial.

Colom, A. (2003) La educación en el contexto de la complejidad: la teoría del caos como paradigma educativo, Revista Española de Pedagogía, 332, 233-248.

Elizalde, A.; MAX-Neef, M. y HopPenhayn, M. (2001) Desarrollo a Escala Humana. Montevideo, Nordan.

Estefanía, J. (2002) Hij@, ¿qué es la globalización? La primera revolución del siglo XXI. Madrid, Santillana Ediciones Generales.

GIDDENS, A. (1994) Consecuencias de la modernidad. Madrid, Alianza.

GIMENO SACRISTÁN, J. (2001) Educar y convivir en la cultura global. Madrid, Morata.

- (2003) Volver a leer la educación desde la ciudadanía, en MARTínez Bonafé, J. Ciudadanía, poder y educación. Barcelona, Graó.

IMBERNON, F. (2002) Cinco ciudadanias para una nueva educación. Barcelona, Graó.

JiMÉNEZ HERRERO, L. (2001) Globalización económica y ecología global, en NOVO, M. y FLoR, J. I. Globalización, crisis ambiental y educación. Madrid, UIMP y MECD.

LóPEZ OSPINA, G. (2001) Medio ambiente, política y sociedad en el siglo XXI, en Novo, M. y FLOR, J. I. Globalización, crisis ambiental y educación. Madrid, UIMP y MECD. 
MAYER, M. (2001) Nuevos retos para la educación ambiental, en Jornadas de Educación Ambiental de Cantabria.

MEADOWs et al. (1972) Los límites del crecimiento. México, Fondo de Cultura Económica.

MELENDRO, M. (2002) Hacia una escuela ambiental y solidaria: ecoauditorías y ecoescuelas, en NOVO, M. y FLOR, J. I. Globalización, crisis ambiental y educación. Madrid, UIMP y MECD.

- (2004) Educación y globalización: educar para la conciencia de los límites, Revista Educación XXI, 6.

MurGA, M. A. (2002) Redes informáticas y educación ambiental, en Novo, M. y FLOR, J. I. Globalización, crisis ambiental y educación. Madrid, UIMP y MECD.

Novo, M. (1995) La educación ambiental. Bases éticas, conceptuales y metodológicas. Madrid, Editorial Universitas.

PREBISCH, R. (1976) Crítica del capitalismo periférico, Revista de la CEPAL, primer semestre de 1976.

ROBERTSON, R. (1992) Globalization. Social Theory and Global Culture. Londres, Sage.

TonuCCI, F. (1997) La Ciudad de los Niños. Madrid, Fundación Germán Sánchez Ruipérez.

TORTOSA, J. M. (2001) El juego global. Barcelona, Icaria.

VILCHES, A. y GIL, D. (2003) Construyamos un futuro sostenible. Madrid, Cambridge University Press.

WADE, D. (1999) Culturas amenazadas, National Geographic, 5 (2), 6-34.

WolTon, D. (2003) L'autre mondialisation. París, Éditions Flammarion.

ZWINGLE, E. (1999) Cultura global, National Geographic, 5 (2), 6-34. 